\title{
Protocolo diagnóstico en fiebre de origen desconocido para países en vías de desarrollo
}

\author{
JESÚS ROMO ${ }^{1}$, JULIÁN MUÑOZ² \\ ${ }^{1}$ Médico Interno de Pregrado, UACJ. Instituto Nacional de Ciencias Médicas y Nutrición \\ "Salvador Zubirán”. México, D. F. ${ }^{2}$ Jefe de Residentes Hospital Universitario \\ "Virgen de las Nieves". Granada, España.
}

\begin{abstract}
Resumen
Signos vitales, como la fiebre, suelen ser motivo de frustración para el binomio médico-paciente; por ello, es indispensable tener en mente una serie de pasos a seguir que nos encaminen al diagnóstico etiológico de esta modalidad patológica. No podemos enfrentar tal padecimiento siguiendo ordenadamente los protocolos recomendados por autores de primer mundo, porque muchas de las veces nos quedamos a mitad del camino, constituyendo con esto una pérdida de tiempo y frustración. Es por eso que surge la necesidad de reorganizar un protocolo bien establecido para ello. En este trabajo, se reorganiza el protocolo diagnóstico establecido por la literatura anglosajona para deducir el factor etiológico en Fiebre de Origen Desconocido (FOD). La mayoría de los autores expertos en el tema recomienda cuatro fases diagnósticas divididas según las herramientas (tecnológicas y científicas) de diagnóstico; la intención de esta revisión es reagrupar la modalidad diagnóstica y que sea de buen provecho para las unidades hospitalarias de América Latina y otros países que están en vías de desarrollo. Presentamos un formato fácil de seguir y que sin duda dará buenos resultados para el rastreo etiológico de este problema.
\end{abstract}

Palabras clave: Fiebre de origen desconocido, etiol; protocolos clínicos; diagnóstico clínico.

\section{Fever of unknown origin diagnostic protocol for developing countries}

\section{Abstract}

Vital signs like the fever usually are frustrating for the binomial doctor-patient; as such it is indispensable to have in mind a series of steps that direct us to the etiologic diagnosis of this pathological modality. We cannot face this disturbance by following protocols recommended by authors of the first world, as many times we remain halfway, constituting a loss of time and frustration. That is why we need to reorganize an established affluent protocol. In this paper, the English diagnosis protocol is reorganized in order to deduce the etiologic factor in Fever of unknown origin (FOD). Most of the authors expert on the subject recommend four divided diagnosis phases according to the tools (technological and scientific) of diagnosis. The intention of this paper is to regroup the diagnosis modality in benefit of hospital units of Latin America and other developing countries. We present a format easy to follow and that without doubt will give good results for the etiology tracking of this problem.

Key words: Fever of unknown origin; clinical protocols; diagnosis, clinical.

Correspondencia:

Dr. Jesús Eduardo Romo

410 Nino Aguilera

El Paso TX 79901, México

E-mail: jesusromo@salud.gob.mx 


\section{INTRODUCCIÓN}

En 1961, Petersdorf y Beeson publicaron una revisión de 100 casos clínicos de pacientes con cuadros de hipertermia de tres semanas de evolución y sin diagnóstico exacto, a pesar de tener una semana de estancia intrahospitalaria ( ${ }^{1}$ y es así como se define el problema de FOD. Poco después surgen opiniones variantes y autores como Durack y Srteet proponen una estancia hospitalaria de 3 días como máximo para no catalogar el factor etiológico $\left({ }^{2}\right)$; factor que sin duda dependerá siempre de las condiciones generales del medio donde se dé el caso. Tenemos que reconocer que una FOD es aún tarea difícil para el médico que se enfrenta ante el paciente con tales características; el antecedente de hipertermia de tres semanas de evolución (como mínimo para establecer grado de cronicidad), sin evidenciar patología en la anamnesis y/o exploración física, así como tampoco detectar anormalidades en estudios de laboratorio y gabinete, sugerirán siempre un síndrome febril de origen desconocido $\left(^{3}\right)$.

La FOD es subcatalogada en cuatro grandes grupos: el tipo clásico, FOD en infección por VIH, fiebre con neutropenia, nosocomial y la episódica recurrente. Existe una gama de posibilidades etiológicas que conllevan al síndrome, como los padecimientos infecciosos (40\% de los casos), neoplasias (20 a 25\%), vasculitis-colágenas $(10$ a $15 \%)$ y misceláneas $(25 \%)^{4-6}$. Si se exceptúa una afección por VIH e infecciones oportunistas, obtendremos un criterio diagnóstico más exacto y diferencial $\left({ }^{4}\right)$.

En países de América Latina, así como en otros que están en vías de desarrollo, se enfrenta el problema de no poder seguir las recomendaciones dadas por los autores anglosajones de manera precisa. Por cuestiones de equipo tecnológico, el diagnóstico etiológico se queda a mitad del camino, constituyendo con esto una pérdida de tiempo y frustración para el médico tratante. Por ello mismo, hemos "renovado" un protocolo dirigido a esta población médica.

\section{PROTOCOLO DIAGNÓSTICO PARA PAÍSES EN VÍAS DE DESARROLLO}

Siempre comenzar realizando un interrogatorio bien dirigido, una exploración física completa y rutinaria, complementados con la evaluación paraclínica dada por los estudios de imagen y laboratorio, que juntos nos encaminarán a realizar el diagnóstico diferencial de las enfermedades, frente a cualquier síndrome patológico. Tratándose de FOD en cualquier modalidad, será conveniente siempre tener un algorritmo que ahorre tiempo y conduzca fácilmente al diagnóstico. Recordar siempre que cada caso es individual y que, apoyados en una evaluación clínica concisa, podremos ubicar mejor la situación del paciente al momento de aplicar el régimen diagnóstico $\left(^{1-5}\right)$.

Comenzar con una entrevista médica de rutina hasta llegar a los estudios más especializados $\left({ }^{7,8}\right)$, y tener en mente las causas más frecuentes de una FOD (infecciones y neoplasias), a ser investigadas en primera instancia $\left({ }^{3,4,6}\right)$. En este trabajo, se propone un protocolo diagnóstico dividido en 5 fases, que abarcan las posibilidades de diagnóstico más adecuadas para unidades de segundo nivel de atención médica; recomendando una evaluación inicial de rutina junto con las órdenes de exámenes paraclínicos (laboratorio y gabinete), continuando con exámenes de mínima invasión, exploraciones altamente invasivas, exámenes de alta especialidad y finalmente dejando la evolución clínica del enfermo para decidir vigilancia ambulatoria o indicar un tratamiento empírico (Figura 1).

\section{Etapa 1: Evaluación clínica básica}

La anamnesis debe ser minuciosa, a fin de completar todos los datos que caracterizen al paciente $\left({ }^{8,9}\right)$. Evaluando delicadamente el padecimiento actual que es el motivo de consulta; no olvidar nunca los antecedentes personales de índole patológico, heredofamiliares, que juegan un papel importante en padecimientos como los neoplásicos. 


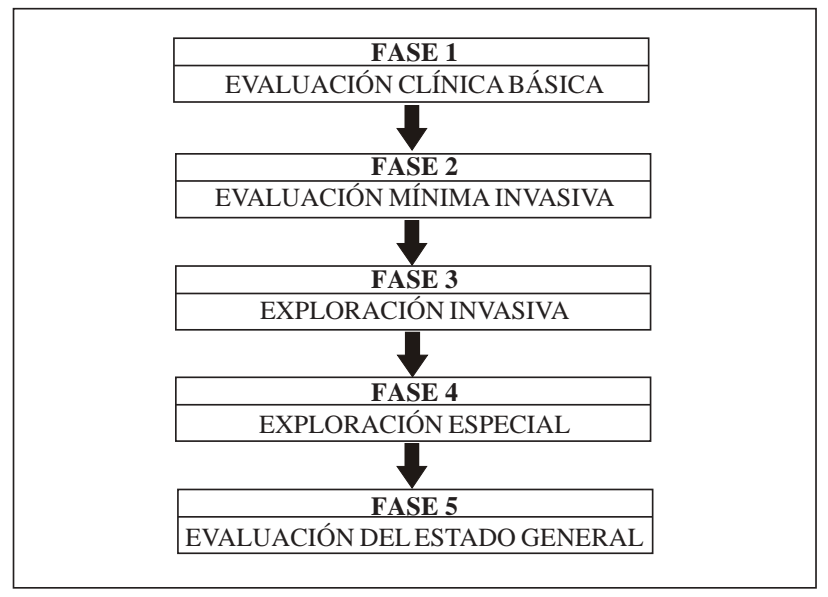

Figura 1.- Fases del protocolo diagnóstico en Fiebre de Origen Desconocido

La exploración física deberá ser completa. Los estudios de gabinete, como la radiografía de tórax, placa simple de abdomen y el electrocardiograma, deben de ser indicados siempre; los estudios de laboratorio en general están encaminados a descartar problemas infecciosos, inmunológicos o metabólicos, por lo que debemos considerar lo más oportuno a primera instancia $\left({ }^{6,9,10}\right)$.

Si a pesar de esta evaluación inicial, no hemos destacado el origen de la fiebre, será conveniente pensar en ordenar nuevos estudios y hacer énfasis en repetir procedimientos exploratorios $\left({ }^{3,4,6,8}\right)$ (Figura 2).

\section{Etapa 2: Evaluación mínima invasiva}

Es conveniente reevaluar el estado del paciente, por medio de una exploración física dirigida a las áreas que nos orienten un nuevo planteamiento, así como repetir estudios de laboratorio e imagen en búsqueda de nuevos datos $\left({ }^{8,9}\right)$. Luego de ello, suspender el uso de fármacos temporalmente, pues pueden enmascarar alguna situación clínica no evidente. Recurrir a exámenes de laboratorio inmunológicos (ANA, anti-ADN, ANCA), estudios micológicos en sangre y orina, parasitología, pruebas endocrinológicas y, de manera especial, las pruebas de función tiroidea; y realizar pruebas de función respiratoria en caso de que se agudice la FOD con un problema respiratorio.

De no encontrar una posibilidad diagnóstica, recurrir a estudios especializados en imagen, descartando patología intestinal por medio de estudios baritados y enema; revisión orgánica a través de tomografía computarizada de tórax y abdomen, recurrir a la IRM si existe sospecha neurológica;solicitar ecocardiografía si la exploración física evidencia un soplo. En caso de sensibilidad al dolor en palpación de senos paranasales, es de utilidad recurrir a radiografía de senos paranasales, así como radiografía sacroiliaca al presentar signos de sensibilidad en esta zona. En mujeres mayores de 35 años, indicar siempre mamografía.

\section{FASE 1. EVALUACIÓN CLÍNICA BÁSICA}

Anamnesis

Ficha de identidad

Motivo de consulta / padecimiento actual

Estado actual de aparatos y sistemas

Antecedentes personales patológicos

Antecedentes personales no patológicos

Antecedentes heredofamiliares

Exploración física completa

Piel y faneras en general

Cabeza: cráneo, órganos de los sentidos, ojos, oídos, nariz, bucofaringe

Tórax: cardiorespiratorio

Abdomen y pelvis

Miembros superiores e inferiores, vascular periférico y columna vertebral

Genitales y perineo

Estudios de laboratorio en general

Básicos: biometría hemática, VSG, bioquímica sanguínea y de orina, PCR, proteinograma, sedimento urinario, hemocultivos y pruebas de función hepática

Serologías: para bacterias como Salmonella y Shigella, viral como CMV, Epstein-Barr y lúes

Otros: sangre oculta en heces, prueba de Mantoux

Estudios de gabinete general

EKG

Rx de tórax

Rx simple de abdomen

Figura 2.- Evaluación clínica básica. 
Si no evidenciamos una posible causa a estas alturas, debemos entonces recurrir a exploraciones meramente invasivas $(3,6,10,11)$ (Figura 3).

\begin{tabular}{|l|}
\multicolumn{1}{|c|}{ FASE 2. EVALUACIÓN MÍNIMA INVASIVA } \\
Rutinario \\
Exploración física minuciosa, según sospecha clínica \\
Repetir Rx de tórax y simple de abdomen \\
Repetir exámenes de laboratorio general \\
Serologías \\
Restricciones \\
Suspender fármacos temporalmente \\
Sin Dx \\
Exámenes de laboratorio especiales \\
ANA, factor reumatoide, anti-DNA, ANC, anti-ENA \\
Micobacterias en sangre y orina \\
Perfiles endocrinológicos en especial las de función toroidea \\
Parasitología \\
Pruebas de función respiratoia \\
$\quad$ Sin Dx \\
Estudios de imagen \\
Tránsito intestinal, enema baritado de colon \\
Tomografía computarizada toracoabdominal y cráneo \\
Resonancia magnética \\
Ultrasonido en abdomen y pelvis \\
Ecocardiografía \\
Rx de senos paranasales y sacroiliacas \\
Mamografía
\end{tabular}

Figura 3.- Evaluación mínima invasiva.

\section{Etapa 3: Exploración invasiva}

Los autores consideran esta tercera etapa como la especializada en estudios de imagen $\left({ }^{4}\right)$, pero hemos observado ya que los estudios radiográficos pueden ser más útiles antes. Continuando con el rastreo diagnóstico de manera invasiva, lo mejor será considerar estudios de imagen, como lo es la gamagrafía, aunque literalmente hay quienes no la consideran invasiva. Cabe la posibilidad de investigar la causa de la enfermedad con procedimientos considerablemente invasivos, como es el caso de la biopsia para investigar médula ósea y piezas hepáticas; están indicadas obviamente cuando la clínica oriente una afección de estos sistemas orgánicos $(3,4,6,8,9,11)$.

Se dejará a última instancia los estudios $100 \%$ invasores, como la endoscopia. Pacientes con afección del tubo digestivo alto (hematemesis, vómito de contenido alimenticio, etc.) es posible indicar una gastroscopia; colonoscopia para aquellos que muestren datos de patología de tubo digestivo bajo; fibrobroncoscopia en quienes muestren anormalidades neumológicas, etc $\left({ }^{8}\right)$ (Figura 4).

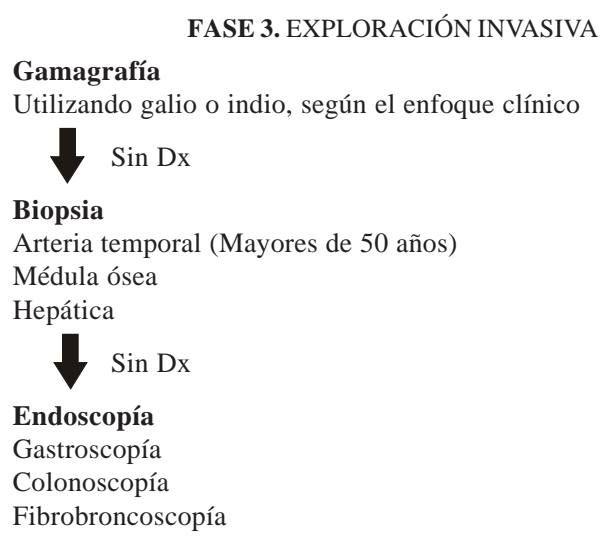

Figura 4.- Exploración invasiva.

\section{Etapa 4: Exploración especial}

Es prudente indicar estudios, como EMG, EEG, punción lumbar, tomografías contrastadas o resonancias magnéticas, por mencionar ejemplos, antes de recurrir a exploraciones invasivas como las que hemos mencionado; pero, se deja a criterio individual sobre este respecto $\left({ }^{8}\right)$. Existen situaciones especiales donde podemos considerar estos exámenes en cuarta fase, como es el caso de pacientes que no hayan presentado antes algún foco con sospecha de neuropatía.

Los estudios angiográficos son de mucha utilidad, siempre apoyados mediante la clínica $\left({ }^{4}\right)$. Los estudios laparoscópicos, técnicamente 
hablando, representan la última herramienta diagnóstica ante una situación como la FOD. Durack y Brusch catalogan este procedimiento como la última etapa en el protocolo diagnóstico de FOD $\left.{ }^{3,6}\right)$ (Figura 5).

\begin{tabular}{|l|}
\hline \multicolumn{1}{|c|}{ FASE 4. EXPLORACIÓN ESPECIAL } \\
EMG, EEG \\
TC de contraste y RM \\
Angiografía \\
Biopsia \\
Punción lumbar \\
Laparoscopía \\
\hline
\end{tabular}

Figura 5.- Exploración especial.

\section{Etapa 5: Evaluación del estado general}

Es básicamente el estudio de la evolución del paciente $\left({ }^{3,8,9}\right)$. En aquellos, quienes a pesar de todos los estudios realizados, estén con un estado general conservado, está indicado seguir el caso de manera ambulatoria $\left({ }^{6}\right)$. A menos que se agudice el padecimiento, se hospitaliza y realiza estudios pertinentes $\left({ }^{4,6}\right)$. En aquellos en quienes se complique su estado general, podemos indicar una laparotomía exploradora y plantear diagnósticos probables.

Ante una duda diagnóstica no dilucidada aún, tengamos presente la posibilidad de infección o neoplasia; está indicado iniciar un tratamiento empírico basado en antibióticos, salicilatos, corticoides o anticancerosos, según el juicio médico (Figura 6).

\section{DISCUSIÓN}

Independientemente del problema patológico, siempre tener en mente y de manera muy clara qué estudios se solicitará primero y posteriormente para manejar nuestro paciente. En quienes presenten FOD, indicar los estudios más básicos, seguidos por los de mediana especialidad y dejar para lo último los de más invasión. En este trabajo, proponemos ajustar algunas normas ya establecidas por los expertos en este padecimiento. Subrayamos que no podemos individualizar las etapas diagnósticas por el tipo de técnica, si no que éstas deben ser catalogadas por el estado clínico del paciente, indicando de primera instancia exámenes como TC, RM, estudios de contraste, etc., si es que el paciente muestra una sintomatología que claramente indique estos estudios, al primer contacto.

\section{Conclusiones:}

Para las unidades hospitalarias de segundo nivel, se debe agotar las posibilidades diagnósticas de la fiebre de origen desconocido encaminadas a la clínica que cada paciente presente; empezando con el estudio base del paciente, como lo es la historia clínica completa y sus estudios paraclínicos de primer nivel. Luego, recomendar el uso de estudios no invasivos y dejar para después los de carácter invasor, siempre y cuando se los justifique con un dato clínico. Si después de todo lo anterior no hemos esclarecido el factor etiológico de la FOD, dejemos que la evolución clínica nos

\begin{tabular}{|ll|}
\hline \multicolumn{2}{|c|}{ FASE 5. EVALUACIÓN DEL ESTADO GENERAL } \\
Estado general conservado \\
Vigilancia ambulatoria \\
Hospitalizar (opcional)
\end{tabular}

Figura 4.- Evaluación del estado general. 
aporte nuevos datos para encontrar la causa de la enfermedad.

\section{REFERENCIAS BIBLIOGRÁFICAS}

1. Petersdorf RO, Beeson PB. Fever of unexplained origin: Report on 100 cases. Medicine (Baltimore) 1961;40:1-30.

2. Durack DT, Street AC. Fever of unknown origin reexamined and redefined. En: Remington JS, Swartz MN (eds). Current Clinical Topics in Infectious Diseases, volume 11. Boston: Blackwell; 1991 p. 35-51.

3. Brusch JL, Weinstein L. Fever of unknown origin. Med Clin North Am 1988;72:1247-61.

4. Lozano de León EM, Gómez-Mateos JM y col. Fiebre de origen desconocido. Actitudes diagnósticas En: Medicine $7^{\text {a }}$ ed. $1998 ; 3558-66$.

5. Moya Mir MS. Fiebre de Origen Desconocido. Frecuencia y metodología diagnóstica. Inflamación-93. 1992; 279-85.

6. Cunha BA. Fever of unknown origin. Infectious Disease Clinics of North America. Philadelphia: WB Saunders Company; 1996.

7. Drenth JPH, Haagsma CJ, Van der Meer JWM, and the International Hyper-IgD Study Group. Hyperimmunoglobulinemia D and periodic fever syndrome: the clinical spectrum in a series of 50 patients. Medicine (Baltimore) 1994;73:133-44.

8. Knockaert DC. Diagnostic strategy for fever of unknown origin in the ultrasonography and computed tomography era. Acta Clin Belg 1992;47:100-16.

9. Gelfand JA, Wolff SM. Fever of unknown origin. En: Mandell GL, Bennett JE, Dolin R, eds. Principles and practice of infectious diseases. $4^{\mathrm{a}}$ ed. New York: Churchill Livingstone; 1995. p. 536-49.
10. Gelfand GA, Dinarello DA, Wolff SM. Fiebre y fiebre de origen desconocido. En: Harrison, ed. Principios de Medicina Interna. 14 ed. New York: Edit. Interamericana; 1996. p. 94-104.

11. Larson EB, Featherstone HJ, Petersdorf RG. Fever of undetermined origin: diagnosis and follow-up of 105 cases. 1970-1980. Medicine (Baltimore) 1982;61:269-92.

12. Barbado FJ, Vázquez JJ, Pena JM, et al. Pyrexia of unknown origin: Changing spectrum of diseases in two consecutives series. Postgrad Med J 1992;68:884.

13. Bissuel F, Leport C, Perrone C, et al. Fever of unknown origin in HIV-infected patients: A critical analysis of a retrospective series of 57 cases. J Intern Med 1993;236:529.

14. Dinarello CA, Wolff SM. Fever of unknown origin. En: Mandel GL, Douglas RG, Bennett JE (eds). Principles and Practice of Infectious Diseases, ed 3. New York: John Wiley and Sons; 1990. p. 468-79.

15. Simon HB, Wolff SM. Granulomatous hepatitis and prolonged fever of unknown origin. A study of 13 patients. Medicine (Baltimore) 1973;52:1-21.

16. Smith JW. Southwestern Internal Medicine Conference: Fever of undetermined origin: Not what it used to be. Am J Med Sci 1986;292:56-64.

17. Petersdof RG. Fever of unknown origin: an old friend revisited. Arch Intern Med 1992;152:21-2.

18. Kanockaert DC, Vanneste LJ, Vanneste SB, Bobbaers HJ. Fever of unknown origin in the 80s: an update of the diagnostic spectrum. Arch Intern Med 1992;152:52-5.

19. Knockaert DC, Vanneste LJ, Bobbaers HJ. Recurrent or episodic fever of unknown origin: review of 45 cases and survey of the literature. Medicine (Baltimore) 1993;72:18496.

20. Aduan RP, Fauci AS, Dale DC, Wolff SM. Prolonged fever of unkown origin (FUO): A prospective study of 347 patients. Clin Res 1978;26:558A (resumen). 